\title{
A New Iterative Method for Suzuki Mappings in Banach Spaces
}

\author{
Junaid Ahmad $\mathbb{D},{ }^{1}$ Kifayat Ullah $\mathbb{D}^{2},{ }^{2}$ Muhammad Arshad, ${ }^{1}$ and Zhenhua Ma $\mathbb{D}^{3}$ \\ ${ }^{1}$ Department of Mathematics, International Islamic University, H-10, Islamabad 44000, Pakistan \\ ${ }^{2}$ Department of Mathematics, University of Science and Technology Bannu, Khyber Pakhtunkhwa 28100, Pakistan \\ ${ }^{3}$ Department of Mathematics and Physics, Hebei University of Architecture, Zhangjiakou 075024, China
}

Correspondence should be addressed to Junaid Ahmad; ahmadjunaid436@gmail.com and Zhenhua Ma; mazhenghua_1981@ 163.com

Received 12 December 2020; Revised 1 February 2021; Accepted 5 February 2021; Published 28 February 2021

Academic Editor: Kaleem R. Kazmi

Copyright (C) 2021 Junaid Ahmad et al. This is an open access article distributed under the Creative Commons Attribution License, which permits unrestricted use, distribution, and reproduction in any medium, provided the original work is properly cited.

In this paper, an efficient new iterative method for approximating the fixed point of Suzuki mappings is proposed. Some important weak and strong convergence results of the proposed iterative method are established in the setting of Banach space. An example illustrates the theoretical outcome.

\section{Introduction and Preliminaries}

Throughout the present research, we shall write $\mathbb{N}$ and $\mathbb{R}$ to denote the set of natural numbers and real numbers set, respectively. We say that a self-map $F$ of a subset $M$ of a Banach space $E=(E,\|\|$.$) is called a contraction map whenever a real$ constant $0 \leq r<1$ exists with the following property:

$$
\|F u-F v\| \leq r\|u-v\|, \quad \text { for all } u, v \in M .
$$

An element $q \in M$ is said to be a fixed point of $F$ provided that $q=F q$. In this manuscript, the notation Fix $(F)$ will throughout denote the fixed point set of $F$. The Banach-Caccioppoli fixed point theorem (see, e.g., $[1,2]$ and others) states that any contraction mapping in the setting of complete metric spaces admits a unique fixed point $q$, and this $q$ is, in fact, the limit of all the sequences $\left\{u_{k}\right\}$ obtained from the Picard iterates [3], that is, $u_{k+1}=F u_{k}$. However, one of the important classes of mappings in fixed point theory is the class of nonexpansive mappings. Notice that, $F$ is called a nonexpansive mapping whenever (1) holds true for $r=1$. In 1965, Browder [4] and Gohde [5] differently proved the same result concerning the existence of fixed points for nonexpansive mappings. Indeed, they suggested that any self-nonexpansive map $F$ of $M$ always admits at least one fixed point whenever $M$ is assumed to be a bounded convex closed subset of some uniformly convex Banach space (UCBS). Nevertheless, the sequence $\left\{u_{k}\right\}$ defined by Picard iterates may not have a limit in the fixed point set associated with a nonexpansive map in general as shown in the next example. Let $M=[0,1]$ and set $F u=1-u$; it is easy to see that $F$ is a self-nonexpansive mapping on $M$ having a unique fixed point $1 / 2$. However, for any $u_{1}=u \neq 1 / 2$, we obtain the sequence of Picard iterates as follows: $u, 1-u, u, 1-u, \ldots$ which is an oscillating sequence and, hence, diverges in $\operatorname{Fix}(F)=\{1 / 2\}$. For providing comparatively better convergence speed and to overcome such situations, different iterative methods have been suggested by some authors (cf. the works of Mann [6], Ishikawa [7], Noor [8], Agarwal et al. [9], Abbas and Nazir [10], Thakur et al. [11], and references therein).

In 2008, Suzuki [12] gained a big break through introducing an interesting extension of nonexpansive mappings as follows. We recall that a self-map $F: M \longrightarrow M$ mapping with $(C)$ property (also called Suzuki mapping) if the following fact is valid:

$$
\begin{aligned}
\frac{1}{2}\|u-F u\| & \leq\|u-v\| \Longrightarrow\|F u-F v\| \\
& \leq\|u-v\|, \quad \text { for each two elements } u, v \in M .
\end{aligned}
$$

One can easily notice that the Suzuki mappings satisfy the nonexpansive requirement for some elements of the domain. Hence, nonexpansive mappings obviously satisfy 
(C) property of Suzuki [12]. Interestingly, an example in [12] (see also an example below) nicely shows that there exist many mappings in the class of Suzuki mappings, which do not belong to the class of nonexpansive mappings. Suzuki also extended the celebrating result of Browder [4] and Gohde [5] from the setting of nonexpansive mappings to the framework of Suzuki mappings.

New iterative methods for the investigation of fixed points and solution of functional equations is the busy research topic and has fruitful applications such as in image recovery and signal processing (see, e.g., [13-19] and others). Therefore, it is our purpose to construct a new iterative method for the larger class of nonexpansive mappings called Suzuki mappings. We also show by an example that this new iterative process gives better approximations as compared to other methods. Suppose $M$ is a closed nonempty convex subset of a given Banach space, and assume further that $\alpha_{k}, \beta_{k}, \gamma_{k} \in(0,1), k \in \mathbb{N}$, and $F$ is a self-map of $M$.

The Mann iterative method [6] is defined as follows:

$$
\left\{\begin{array}{l}
u_{1}=u \in M, \\
u_{k+1}=\left(1-\alpha_{k}\right) u_{k}+\alpha_{k} F u_{k} .
\end{array}\right.
$$

The Ishikawa [7] iterative method is the extension of the Mann method from one step to two steps as follows:

$$
\left\{\begin{array}{l}
u_{1}=u \in M \\
v_{k}=\left(1-\beta_{k}\right) u_{k}+\beta_{k} F u_{k}, \\
u_{k+1}=\left(1-\alpha_{k}\right) u_{k}+\alpha_{k} F v_{n}
\end{array}\right.
$$

The Noor [8] iterative method is the extension of both of the Mann and Ishikawa iterative methods as follows:

$$
\left\{\begin{array}{l}
u_{1}=u \in M \\
w_{k}=\left(1-\gamma_{k}\right) u_{k}+\gamma_{k} F u_{k} \\
v_{k}=\left(1-\beta_{k}\right) u_{k}+\beta_{k} F w_{k} \\
u_{k+1}=\left(1-\alpha_{k}\right) u_{k}+\alpha_{n} F v_{k} .
\end{array}\right.
$$

Agarwal et al.'s [9] method is the slightly modification of the Ishikawa method as follows:

$$
\left\{\begin{array}{l}
u_{1}=u \in M, \\
v_{k}=\left(1-\beta_{k}\right) u_{k}+\beta_{k} F u_{k}, \\
u_{k+1}=\left(1-\alpha_{k}\right) F u_{k}+\alpha_{k} F v_{n}
\end{array}\right.
$$

Abbas and Nazir's [10] iterative method is a three-step method read as follows:

$$
\left\{\begin{array}{l}
u_{1}=u \in M, \\
w_{k}=\left(1-\gamma_{k}\right) u_{k}+\gamma_{k} F u_{k}, \\
v_{k}=\left(1-\beta_{k}\right) F u_{k}+\beta_{k} F w_{k}, \\
x_{k+1}=\left(1-\alpha_{k}\right) F v_{k}+\alpha_{k} F w_{k} .
\end{array}\right.
$$

Thakur et al. [11] proposed a new iterative method as follows:

$$
\left\{\begin{array}{l}
u_{1}=u \in M, \\
w_{k}=\left(1-\beta_{k}\right) u_{k}+\beta_{k} F u_{k}, \\
v_{k}=F\left(\left(1-\alpha_{k}\right) u_{k}+\alpha_{k} w_{k}\right), \\
u_{k+1}=F v_{k} .
\end{array}\right.
$$

Thakur et al. [11] showed that method (8) is better than all of the methods, namely, Mann (3), Ishikawa (4), Noor (5), Agarwal (6), and Abbas and Nazir (7). Here, in the current research, we first suggest an efficient new iterative method and prove that it can be used for computations of fixed points of the larger class of nonexpansive maps called Suzuki maps. Furthermore, we shall provide a novel example of the so-called Suzuki mappings and prove that it exceeds the corresponding class of nonexpansive mappings.

\section{Preliminaries}

Here, first we present some earlier important definitions, which are needed for our theoretical outcome.

Let $E$ be a given Banach space, and suppose $\left\{u_{k}\right\} \subseteq E$ is weakly convergent to $w \in E$ and satisfies the following:

$$
\liminf _{k \longrightarrow \infty}\left\|u_{k}-w\right\|<\liminf _{k \longrightarrow \infty}\left\|u_{k}-z\right\|, \quad \text { for all } z \in E-\{w\} .
$$

Whenever any weakly convergent sequence in $E$ has the abovementioned property, $E$ is called a Banach space endowed with Opial's property (for details, see [20]). We now recall a property $I$ introduced by Sentor and Dotson [21] for $F: M \longrightarrow M$ (where $M$ is a nonempty subset of a Banach space). We recall that $F$ has condition $I$ [21] if one can find a nondecreasing function, namely, $P:[0, \infty) \longrightarrow[0, \infty)$, with the properties $P(0)=0$, $P(a)>0$ for every $a>0$, and $\|u-F u\| \geq P(\operatorname{dist}(u, \operatorname{Fix}(F)))$ for all $u \in M$.

Let $M$ be any nonempty subset of a general Banach space $E$, and suppose $\left\{u_{k}\right\}$ is any given bounded sequence in $E$. We fix $u \in E$ and denote

(a) by $\mathscr{R}\left(u,\left\{u_{k}\right\}\right)$, the asymptotic radius of $\left\{u_{k}\right\}$ at $u$ given by $\mathscr{R}\left(u,\left\{u_{k}\right\}\right):=\limsup _{k \rightarrow \infty}\left\|u-u_{k}\right\|$

(b) by $\mathscr{R}\left(M,\left\{u_{k}\right\}\right)$, the asymptotic radius associated with $M$ of $\left\{u_{k}\right\}$ given by $\mathscr{R}\left(M,\left\{u_{k}\right\}\right)=\inf \left\{\mathscr{R}\left(u,\left\{u_{k}\right\}\right): u \in M\right\}$

(c) by $\mathscr{Z}\left(M,\left\{u_{k}\right\}\right)$, the asymptotic center associated with $M \quad$ of $\quad\left\{u_{k}\right\} \quad$ given by
$\mathscr{Z}\left(M,\left\{u_{k}\right\}\right)=\left\{u \in E: \mathscr{R}\left(u,\left\{u_{k}\right\}\right)=\mathscr{R}\left(M,\left\{u_{k}\right\}\right)\right\}$

The most well-known fact about the set $\mathscr{Z}\left(M,\left\{u_{k}\right\}\right)$ is that it is always singleton whenever $X$ is UCBS [22]. The fact that the set $\mathscr{Z}\left(M,\left\{u_{k}\right\}\right)$ is convex nonempty whenever $M$ is weakly compact and convex is also well known (see, e.g., $[23,24])$.

Lemma 1 (See [12]). Assume that $M$ is any nonempty subset of a Banach space, and suppose $F: M \longrightarrow M$. If $F$ is a Suzuki mapping, then for every element $u \in M$ and for every element $q \in \operatorname{Fix}(F)$, the fact $\|F u-F q\| \leq\|u-q\|$ holds. 
Lemma 2 (See [12]). Assume that $M$ is any nonempty subset of a Banach space, and suppose F: $M \longrightarrow M$. If F is a Suzuki mapping, then for every two elements $u, v \in M$, we have the following property:

$$
\|u-F v\| \leq 3\|u-F u\|+\|u-v\| .
$$

The following result is known as the demiclosed principle.

Lemma 3 (See [12]). Assume that $M$ is any nonempty subset of a Banach space having the Opial property, and suppose $F: M \longrightarrow M$. If $F$ is a Suzuki mapping, then the following condition holds:

$$
\left\{u_{k}\right\} \subseteq M, \quad u_{k}-w,\left\|u_{k}-F u_{k}\right\| \longrightarrow 0 \Rightarrow F w=w .
$$

The fixed-point set endowed with a Suzuki mapping enjoys the following properties.

Lemma 4 (See [12]). Assume that $M$ is any nonempty subset of a Banach space, and suppose F: $M \longrightarrow M$. If $F$ is a Suzuki mapping, then the set Fix $(F)$ is closed. Furthermore, if $E$ is a strictly convex Banach space and $M$ is convex, then Fix $(F)$ is convex too.

The following useful lemma can be found in [25].

Lemma 5. Let $0<u \leq \gamma_{k} \leq v<1$ for each $k \in \mathbb{N}$ and $\eta \geq 0$. If $\left\{y_{k}\right\}$ and $\left\{z_{k}\right\}$ are any sequences in a UCBS, $E$ with $\limsup _{k \rightarrow \infty}\left\|y_{k}\right\| \leq \eta, \quad \limsup _{k \rightarrow \infty}\left\|z_{k}\right\| \leq \eta, \quad$ and $\lim _{k \rightarrow \infty}\left\|\gamma_{k} y_{k}+\left(1-\gamma_{k}\right) z_{k}\right\|=\eta$.

Then, $\lim _{k \rightarrow \infty}\left\|y_{k}-z_{k}\right\|=0$.

\section{Main Results}

Strongly motivated by those mentioned above, we introduce a new iterative process, namely, JK iteration, as follows:

$$
\left\{\begin{array}{l}
u_{1}=u \in M, \\
w_{k}=\left(1-\beta_{k}\right) u_{k}+\beta_{k} F u_{k}, \\
v_{k}=F w_{k}, \\
u_{k+1}=F\left(\left(1-\alpha_{k}\right) F w_{k}+\alpha_{k} F v_{k}\right), \quad k \in \mathbb{N},
\end{array}\right.
$$

where $\alpha_{k}, \beta_{k} \in(0,1)$.

In the present research section, we establish very interesting and important results for the larger class of the socalled Suzuki maps under the newly suggested method (12). We will present a numerical example to show that the JK iterative process is better than the iterative process by Thakur et al. (8). Furthermore, in the last section, a novel example of the so-called Suzuki maps which is not nonexpansive shows that Suzuki maps properly include nonexpansive maps. The numerical observations suggest that JK iterative method is for better than the leading method of Thakur and, hence, many others.
We now state and prove a much needed lemma for our main outcome, which will play a significant role in each result of the sequel.

Lemma 6. Assume that $M$ is any nonempty closed convex subset of a Banach space $X$, and suppose $F: M \longrightarrow M$ is a Suzuki mapping with Fix $(F) \neq \varnothing$. Suppose $\left\{u_{k}\right\}$ is a sequence given in (12). Then, $\lim _{k \rightarrow \infty}\left\|u_{k}-q\right\|$ exists for every fixed point $q$ of $F$.

Proof. Take $q \in \operatorname{Fix}(F)$. By Lemma 1, we have

$$
\begin{aligned}
\left\|w_{k}-q\right\| & =\left\|\left(1-\beta_{k}\right) u_{k}+\beta_{k} F u_{k}-q\right\| \\
& \leq\left(1-\beta_{k}\right)\left\|u_{k}-q\right\|+\beta_{k}\left\|F u_{k}-q\right\| \\
& \leq\left(1-\beta_{k}\right)\left\|u_{k}-q\right\|+\beta_{k}\left\|u_{k}-q\right\| \\
& \leq\left\|u_{k}-q\right\|, \\
\left\|v_{k}-q\right\| & =\left\|F w_{k}-q\right\| \leq\left\|w_{k}-q\right\| .
\end{aligned}
$$

They imply that

$$
\begin{aligned}
\left\|u_{k+1}-q\right\| & =\left\|F\left(\left(1-\alpha_{k}\right) F w_{k}+\alpha_{k} F v_{k}\right)-q\right\| \\
& \leq\left\|\left(1-\alpha_{k}\right) F w_{k}+\alpha_{k} F y_{k}-q\right\| \\
& \leq\left(1-\alpha_{k}\right)\left\|F w_{k}-q\right\|+\alpha_{k}\left\|F v_{k}-q\right\| \\
& \leq\left(1-\alpha_{k}\right)\left\|w_{k}-q\right\|+\alpha_{k}\left\|v_{k}-q\right\| \\
& \leq\left(1-\alpha_{k}\right)\left\|w_{k}-q\right\|+\alpha_{k}\left\|w_{k}-q\right\|, \\
& =\left\|w_{k}-q\right\| \leq\left\|u_{k}-q\right\| .
\end{aligned}
$$

From the equations mentioned above, we conclude that $\left\{\left\|u_{k}-q\right\|\right\}$ is a bounded and nonincreasing sequence of reals, and hence, $\lim _{k \rightarrow \infty}\left\|u_{k}-q\right\|$ exists for every fixed point $q$ of F.

Theorem 1. Assume that $M$ is any nonempty closed convex subset of a UCBC, and suppose $F: M \longrightarrow M$ is a Suzuki mapping. Assume further that $\left\{u_{k}\right\}$ is a sequence given in (12). Then, $\operatorname{Fix}(F) \neq \varnothing$ if and only if $\left\{u_{k}\right\}$ is bounded, and $\lim _{k \rightarrow \infty}\left\|F u_{k}-u_{k}\right\|=0$.

Proof. First, we assume that $\left\{u_{k}\right\}$ is bounded and $\lim _{k \rightarrow \infty}\left\|F u_{k}-u_{k}\right\|=0$. We shall prove that $\operatorname{Fix}(F) \neq \varnothing$. For this, let $q \in \mathscr{Z}\left(M,\left\{u_{k}\right\}\right)$. By Lemma 2 , we have

$$
\begin{aligned}
\mathscr{R}\left(F q,\left\{u_{k}\right\}\right) & =\underset{k \longrightarrow \infty}{\limsup }\left\|u_{k}-F q\right\| \\
\leq & \underset{k \longrightarrow \infty}{\limsup }\left\|u_{k}-F u_{k}\right\|+\underset{k \longrightarrow \infty}{\limsup }\left\|u_{k}-q\right\|, \\
& =\underset{k \longrightarrow \infty}{\limsup }\left\|u_{k}-q\right\|, \\
& =\mathscr{R}\left(q,\left\{u_{k}\right\}\right) .
\end{aligned}
$$

It follows that $F q \in \mathscr{Z}\left(M,\left\{u_{k}\right\}\right)$. Since in UCBS, asymptotic centers are singleton, we have $F q=q$. Hence, the fixed point is nonempty. 
Conversely, we assume that $\operatorname{Fix}(F) \neq \varnothing$. Conclusions of Lemma 6 provide that $\left\{u_{k}\right\}$ is bounded and $\lim _{k \rightarrow \infty}\left\|u_{k}-q\right\|$ exists for every fixed point $q$ of $F$. Now, if

$$
\lim _{n \rightarrow \infty}\left\|u_{k}-q\right\|=\eta
$$

then by observing the proof of Lemma 6 and keeping (16) in mind, we obtain

$$
\underset{k \longrightarrow \infty}{\limsup }\left\|w_{k}-q\right\| \leq \underset{n \longrightarrow \infty}{\limsup }\left\|u_{k}-q\right\|=\eta .
$$

Appling Lemma 1, we get

$$
\underset{k \longrightarrow \infty}{\limsup }\left\|F u_{k}-q\right\| \leq \underset{k \longrightarrow \infty}{\limsup }\left\|u_{k}-q\right\|=\eta,
$$

and by observing the proof of Lemma 6 , we see

$$
\left\|u_{k+1}-q\right\| \leq\left\|w_{k}-q\right\| \text {. }
$$

It gives, together with (16),

$$
\eta \leq \liminf _{k \longrightarrow \infty}\left\|w_{k}-q\right\| .
$$

From (17) and (20), we obtain

$$
\eta=\lim _{k \longrightarrow \infty}\left\|w_{k}-q\right\| \text {. }
$$

From (21), we have

$$
\begin{aligned}
\eta & =\lim _{k \longrightarrow \infty}\left\|w_{k}-q\right\|, \\
& =\lim _{k \longrightarrow \infty}\left\|\left(1-\beta_{k}\right) u_{k}+\beta_{k} F u_{k}-q\right\|, \\
& =\lim _{k \rightarrow \infty}\left\|\left(1-\beta_{k}\right)\left(u_{k}-q\right)+\beta_{k}\left(F u_{k}-q\right)\right\| .
\end{aligned}
$$

Hence,

$$
\eta=\lim _{k \longrightarrow \infty}\left\|\left(1-\beta_{k}\right)\left(u_{k}-q\right)+\beta_{k}\left(F u_{k}-q\right)\right\| .
$$

Now, from (16), (18) and (23) together with Lemma 5, we obtain

$$
\lim _{k \longrightarrow \infty}\left\|F u_{k}-u_{k}\right\|=0
$$

Now, we are in the position to prove our weak convergence result.

Theorem 2. Assume that E is a UCBS with Opial's property and $M$ is a nonempty convex closed subset of $E$, and suppose $F: M \longrightarrow M$ be a Suzuki mapping with Fix $(F) \neq \varnothing$. Suppose $\left\{u_{k}\right\}$ is a sequence given in (12). Then, $\left\{u_{k}\right\}$ converges weakly to a fixed point of $F$.

Proof. By Theorem 1, $\left\{u_{k}\right\}$ is bounded and $\lim _{k \rightarrow \infty}\left\|F u_{k}-u_{k}\right\|=0$. Since $E$ is uniformly convex, $E$ is reflexive. Hence, one can easily find a subsequence, namely, $\left\{u_{k_{l}}\right\}$ of $\left\{u_{k}\right\}$ such that $u_{k_{l}} \rightarrow u$ for some $u \in M$. By Lemma 3 , $u \in \operatorname{Fix}(F)$. We shall prove that $u$ is the weak limit of $\left\{u_{k}\right\}$. Let $u$ not be the weak limit of $\left\{u_{k}\right\}$. Then, one can find another subsequence, namely, $\left\{u_{k_{m}}\right\}$ of $\left\{u_{k}\right\}$ such that $u_{k_{m}} \rightarrow u$ and $v \neq u$. Again by Lemma 3, $v \in \operatorname{Fix}(F)$. Now, using Lemma 6 and Opial's property, we have

$$
\begin{aligned}
\lim _{k \rightarrow \infty}\left\|u_{k}-u\right\| & =\lim _{l \longrightarrow \infty}\left\|u_{k_{l}}-u\right\| \\
& <\lim _{l \longrightarrow \infty}\left\|u_{k_{l}}-v\right\|, \\
& =\lim _{n \longrightarrow \infty}\left\|u_{k}-v\right\|, \\
& =\lim _{m \longrightarrow \infty}\left\|u_{k_{m}}-v\right\| \\
& <\lim _{m \longrightarrow \infty}\left\|u_{k_{m}}-u\right\|, \\
& =\lim _{n \longrightarrow \infty}\left\|u_{k}-u\right\| .
\end{aligned}
$$

Hence, $\lim _{k \longrightarrow \infty}\left\|u_{k}-u\right\| \leq \lim _{k \longrightarrow \infty}\left\|u_{k}-v\right\|$ by $\lim _{n \longrightarrow \infty}$ $\left\|u_{k}-u\right\|<\lim _{k \rightarrow \infty}\left\|u_{k}-v\right\|$, clearly a contradiction, and so we must accept that $u$ is the only weak limit of $\left\{u_{k}\right\}$.

Now, we prove the following strong convergence result.

Theorem 3. Assume that $M$ is any nonempty convex compact subset of a UCBC, and suppose $F: M \longrightarrow M$ be a Suzuki mapping. Assume further that $\left\{u_{k}\right\}$ is a sequence given in (12). Then, $\left\{u_{k}\right\}$ converges strongly to a fixed point of $F$.

Proof. From Theorem in [12], we can write $\operatorname{Fix}(F) \neq \varnothing$. By Theorem $1, \lim _{k \rightarrow \infty}\left\|F u_{k}-u_{k}\right\|=0$. Since the domain $M$ is compact, one can easily find a strongly convergent subsequence, namely, $\left\{u_{k_{j}}\right\}$ of $\left\{u_{k}\right\}$ having a limit say $z$. By using Lemma 2, the following holds:

$$
\left\|u_{k_{j}}-F z\right\| \leq 3\left\|u_{k_{j}}-F u_{k_{j}}\right\|+\left\|u_{k_{j}}-z\right\|
$$

Hence, $u_{k_{\dot{j}}} \longrightarrow F z$ whenever $j \longrightarrow \infty$, so the uniqueness of limits follows $F z=z$. By Lemma 6, $\lim _{k \rightarrow \infty}\left\|u_{k}-z\right\|$ exists. Hence, $z$ is the strong limit of $\left\{u_{k}\right\}$.

The proof of the following theorem is elementary and, therefore, omitted.

Theorem 4. Assume that $M$ is any nonempty closed convex subset of a UCBS, and suppose $F: M \longrightarrow M$ be a Suzuki mapping. If Fix $(F) \neq \varnothing$ and $\liminf _{k \rightarrow \infty} \operatorname{dist}\left(u_{k}\right.$, Fix $\left.(F)\right)=$ 0 , where $\left\{u_{k}\right\}$ is a sequence given in (12), then $\left\{u_{k}\right\}$ converges strongly to a fixed point of $F$.

We finish this section with a strong convergence theorem under the condition $I$.

Theorem 5. Assume that $M$ is any nonempty convex closed subset of a UCBS, and suppose $F: M \longrightarrow M$ be a Suzuki mapping with Fix $(F) \neq \varnothing$. Assume further that $\left\{u_{k}\right\}$ is a sequence given in (12). If $F$ fulfils condition (I), then $\left\{u_{k}\right\}$ converges strongly to a fixed point of $F$.

Proof. In view of Theorem 1, we can conclude that $\lim \inf _{k \rightarrow \infty}\left\|F u_{k}-u_{k}\right\|=0$. Since $F$ fulfils condition $(I)$, one has $\liminf \operatorname{in}_{k \rightarrow \infty} \operatorname{dist}\left(u_{k}, \operatorname{Fix}(F)\right)=0$. The conclusions are now clear from Theorem 4. 
TABLE 1: Sequences defined by JK, Thakur, and Agarwal iterative methods for mapping $F$ defined in Example 1.

\begin{tabular}{lccc}
\hline$k$ & JK $(12)$ & Thakur $(8)$ & Agarwal (6) \\
\hline 1 & 0.9 & 0.9 & 0.9 \\
2 & 0.9982071573 & 0.9965059644 & 0.9825298221 \\
3 & 0.9999814317 & 0.9998861029 & 0.9971525733 \\
4 & 0.9999999265 & 0.9999964422 & 0.9995552826 \\
5 & 1 & 0.9999998920 & 0.9999325257 \\
6 & 1 & 0.9999999967 & 0.9999899750 \\
7 & 1 & 0.9999999999 & 0.9999985340 \\
8 & 1 & 1 & 0.9999997883 \\
9 & 1 & 1 & 0.9999999697 \\
10 & 1 & 1 & 0.9999999957 \\
11 & 1 & 1 & 0.9999999994 \\
12 & 1 & 1 & 0.9999999999 \\
13 & 1 & 1 & $\mathbf{1}$ \\
\hline
\end{tabular}

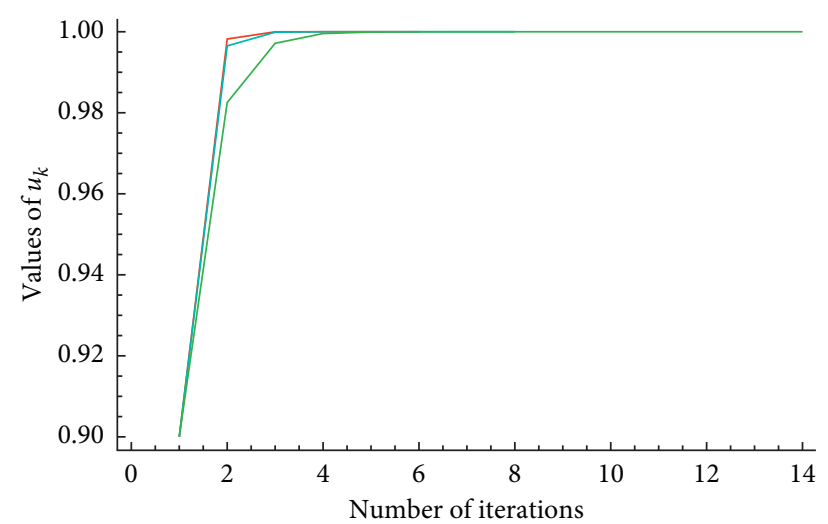

Figure 1: Convergence behavior of JK (12), Thakur (8), and Agarwal (6) iterates to converge to $q=1$, a unique fixed point of the self-map $F$ in Example 1 where $u_{1}=0.9$.

TABLE $2: \alpha_{k}=((k+1) /(5 k+1))^{1 / 11}$ and $\beta_{k}=k / \sqrt{2 k+3}$

\begin{tabular}{lccc}
\hline \multicolumn{2}{l}{ Iterates number to converge an iteration to 1} & & Thakur \\
$u_{1}$ & $\mathrm{~S}$ & 11 & $\mathbf{8}$ \\
\hline 0.10 & 20 & 11 & $\mathbf{8}$ \\
0.15 & 20 & 11 & $\mathbf{8}$ \\
0.20 & 20 & 10 & $\mathbf{8}$ \\
0.50 & 20 & 10 & $\mathbf{8}$ \\
0.80 & 19 & 10 & $\mathbf{7}$ \\
0.90 & 19 & & \\
\hline
\end{tabular}

TABLE 3: $\alpha_{k}=k /(6 k+7)$ and $\beta_{k}=1 /(2 k+3)^{1 / 5}$.

Iterates number to converge an iteration to 1

\begin{tabular}{lccc}
$u_{1}$ & $\mathrm{~S}$ & Thakur & JK \\
\hline 0.10 & 21 & 11 & $\mathbf{9}$ \\
0.15 & 21 & 11 & $\mathbf{9}$ \\
0.20 & 21 & 11 & $\mathbf{9}$ \\
0.50 & 21 & 11 & $\mathbf{9}$ \\
0.80 & 21 & 11 & $\mathbf{9}$ \\
0.90 & 20 & 10 & $\mathbf{9}$ \\
\hline
\end{tabular}


TABLE 4: $\alpha_{k}=\sqrt{k} /(k+5)^{1 / 7}$ and $\beta_{k}=\sqrt{1 /(7 k+3)}$.

Iterates number to converge an iteration to 1

\begin{tabular}{lccc}
$u_{1}$ & $\mathrm{~S}$ & Thakur & $\mathrm{JK}$ \\
\hline 0.10 & 19 & 11 & 7 \\
0.15 & 19 & 11 & 7 \\
0.20 & 19 & 11 & 7 \\
0.50 & 19 & 11 & 7 \\
0.80 & 19 & 10 & 7 \\
0.90 & 19 & 10 & 7 \\
\hline
\end{tabular}

\section{Numerical Example}

This section introduces a novel example of self-Suzuki maps on a closed convex bounded subset of a Banach space. We suggest with many different cases that the novel JK scheme is far better than the earlier iterative methods using this example. Since we are using Suzuki maps in our work, the provided outcome holds simultaneously for nonexpansive maps as well.

Example 1. Consider a closed convex $M=[0,1]$ of a Banach space $\mathbb{R}$. Set a self-map $F: M \longrightarrow M$ as follows:

$$
F u= \begin{cases}1-u, & \text { if } u<\frac{1}{8}, \\ \frac{u+4}{5}, & \text { if } u \geq \frac{1}{8} .\end{cases}
$$

One can conclude that $F$ is a Suzuki mapping and, however, not nonexpansive by studying the computations given below. Select $u=8 / 65$ and $v=1 / 8$, and observe that

$$
\begin{aligned}
\|F u-F v\| & =|F u-F v|=\left|1-\frac{8}{65}-\frac{33}{40}\right|, \\
& =\frac{135}{2600}>\frac{1}{520}=\|u-v\|,
\end{aligned}
$$

which proves that $F$ is not a nonexpansive on $M$.

Next, we suggest the proof of the Suzuki property of $F$ on $M$. The proof can be divided as given below.

Case 1. Select $u \in[0,(1 / 8))$; then, $(1 / 2]\|u-v\|=((1-2 u) /$ $2) \in((3 / 8),(1 / 2))$. For $(1 / 2)\|u-F u\| \leq\|u-v\|$, one has $((1-2 u) / 2) \leq v-u$, i.e., $(1 / 2) \leq v$; therefore, $v \in[(1 / 2), 1]$. So, one has

$$
\begin{gathered}
\|F u-F v\|=\left|\frac{v+4}{5}-(1-u)\right|=\left|\frac{v+5 u-1}{5}\right|<\frac{1}{8}, \\
\|u-v\|=|u-v|>\left|\frac{1}{8}-\frac{1}{2}\right|=\frac{3}{8} .
\end{gathered}
$$

Hence, $(1 / 2)\|u-F u\| \leq\|u-v\| \Rightarrow\|F u-F v\| \leq\|u-v\|$.

Case 2. Select $u \in[(1 / 8), 1] ; \quad$ then, $\quad(1 / 2) \| u-$ $F u \|=(1 / 2)|((u+4) / 5)-u|=((4-4 u) / 10) \in[0,(7 / 20)]$. For $(1 / 2)\|u-F u\| \leq\|u-v\|$, one has $((4-4 u) / 10) \leq|v-u|$, and so the following possible situations occur: (a) Whenever $u<v, \quad((4-4 u) / 10) \leq v-u \Rightarrow v \geq((4+6 u) /$ $10) \Rightarrow v \in[(19 / 40), 1] \subset[(1 / 8), 1]$. So,

$$
\|F u-F v\|=\left|\frac{u+4}{5}-\frac{v+4}{5}\right|=\frac{1}{5}\|u-v\| \leq\|u-v\| .
$$

Therefore, $\quad(1 / 2)\|u-F u\| \leq\|u-v\| \Rightarrow\|F u-F v\| \leq$ $\|u-v\|$.

(b) Whenever $u>v,((4-4 u) / 10) \leq u-v \Rightarrow v \leq u-((4-$ $4 u) / 10)=((14 u-4) / 10) \Rightarrow v \in[-(9 / 40), 1]$. Since $v \in[0,1], v \leq((14 u-4) / 10) \Rightarrow u \in[(4 / 14), 1]$. So, the case is $u \in[(4 / 14), 1]$ and $v \in[0,1]$.

When $u \in[(4 / 14), 1]$ and $v \in[(1 / 8), 1]$ are already included in $(a)$, we assume $u \in[(4 / 14), 1]$ and $v \in[0,(1 / 8))$; then,

$$
\begin{aligned}
\|F u-F v\| & =\left|\frac{u+4}{5}-(1-v)\right|, \\
& =\left|\frac{u+5 v-1}{5}\right| .
\end{aligned}
$$

For convenience, first we assume $u \in[(4 / 14),(1 / 2)]$ and $v \in[0,(1 / 8)), \quad$ then $\quad\|F u-F v\| \leq(1 / 8) \quad$ and $\|u-v\|>(18 / 112)$. Hence, $\|F u-F v\| \leq\|u-v\|$.

Next, we consider $u \in[(1 / 2), 1]$ and $v \in[0,(1 / 8))$, then $\|F u-F v\| \leq(1 / 8) \quad$ and $\quad\|u-v\|>(3 / 8)$. Hence, $\|F u-F v\| \leq\|u-v\| . \quad$ So, $\quad(1 / 2)\|u-F u\| \leq\|u-v\| \Rightarrow$ $\|F u-F v\| \leq\|u-v\|$. Hence, $F$ is a Suzuki mapping on $M$. Let $\alpha_{k}=2 k /(7 k+9)$ and $\beta_{k}=(1 /(3 k+7))^{1 / 2}, k \in \mathbb{N}$. A strong convergence of JK (12), leading Thakur (8), and leading twostep Agarwal (6) iterates to $q=1$ can be seen in the Table 1 and Figure 1. Clearly, JK iterates converge faster to $q=1$.

Now, to show further effectiveness of JK iteration, we set stopping criteria $\left\|u_{k}-q\right\|<10^{-15}$, where $q=1$ is a fixed point of $F$. The observations are given below.

Remark 1. One can observe from Tables 1-4 that the new JK iterative method converges faster than the fastest Thakur and fastest two-step Agarwal methods for the larger class of Suzuki mappings.

\section{Data Availability}

No data were used to support this study.

\section{Conflicts of Interest}

The authors declare that they have no conflicts of interest.

\section{Authors' Contributions}

J. A., K. U., M. A., and Z. M. provided equal contributions to this article. 


\section{Acknowledgments}

This research was funded by Nature Science Foundation of Heibei Province under Grant A2019404009, Postdoctoral Foundation of Heibei Province under Grant B2019003016, Innovation and Improvement Project of Academic Team of Hebei University of Architecture (Mathematics and Applied Mathematics) NO. TD202006, and Major Project of Hebei Provincial Department of Education of 2021.

\section{References}

[1] S. Banach, "Sur les opérations dans les ensembles abstraits et leur application aux équations intégrales," Fundamenta Mathematicae, vol. 3, p. 133, 1922.

[2] R. Caccioppoli, "Un teorema generale sull'esistenza di elementi uniti in una transformazione funzionale," Rendiconti Accademy Lincei, vol. 11, pp. 794-799, 1930.

[3] E. Picard, "Memoire sur la theorie des equations aux derivees partielles et la methode des approximations successives," Journal de Mathématiques Pures et Appliquées, vol. 6, p. 145, Article ID 210, 1890.

[4] F. E. Browder, "Nonexpansive nonlinear operators in a Banach space," Proceedings of the National Academy of Sciences, vol. 54, no. 4, p. 1041, 1965.

[5] D. Göhde, "Zum prinzip der Kontraktiven abbildung," Mathematische Nachrichten, vol. 30, no. 3-4, p. 251, 1965.

[6] W. R. Mann, "Mean value methods in iteration," Proceedings of the American Mathematical Society, vol. 4, no. 3, pp. 506-510, 1953.

[7] S. Ishikawa, "Fixed points by a new iteration method," Proceedings of the American Mathematical Society, vol. 44, no. 1, p. 147, 1974.

[8] M. A. Noor, "New approximation schemes for general variational inequalities," Journal of Mathematical Analysis and Applications, vol. 251, no. 1, pp. 217-229, 2000.

[9] R. P. Agarwal, D. O’Regan, and D. R. Sahu, "Iterative construction of fixed points of nearly asymptotically nonexpansive mappings," Journal of Nonlinear and Convex Analysis, vol. 8, no. 1, pp. 61-79, 2007.

[10] A. Abbas and T. Nazir, "A new faster iteration process applied to constrained minimization and feasibility problems," Matematički Vesnik, vol. 66, pp. 223-234, 2014.

[11] B. S. Thakur, D. Thakur, and M. Postolache, "A new iterative scheme for numerical reckoning fixed points of Suzuki's generalized nonexpansive mappings," Applied Mathematics and Computation, vol. 275, p. 147, 2016.

[12] T. Suzuki, "Fixed point theorems and convergence theorems for some generalized nonexpansive mappings," Journal of Mathematical Analysis and Applications, vol. 340, no. 2, p. $1088,2008$.

[13] C. Byrne, "A unified treatment of some iterative algorithms in signal processing and image reconstruction," Inverse Problems, vol. 20, no. 1, p. 103, 2004.

[14] C. I. Podilchuk and R. J. Mammone, "Image recovery by convex projections using a least-squares constraint," Journal of the Optical Society of America A, vol. 7, no. 3, p. 517, 1990.

[15] D. C. Youla, "On deterministic convergence of iterations of relaxed projection operators," Journal of Visual Communication and Image Representation, vol. 1, no. 1, p. 12, 1990.

[16] J. Ali and F. Ali, "Approximation of common fixed points and the solution of image recovery problem," Results Math, vol. 74, pp. 1-22, 2019.
[17] M. Hasanuzzaman, M. Imdad, and M. Imdad, "Relation theoretic metrical fixed point results for Suzuki type ZRcontraction with an application," AIMS Mathematics, vol. 5, no. 3, p. 2071, 2020.

[18] M. Ahmadullah, J. Ali, and M. Imdad, "Unified relationtheoretic metrical fixed point theorems under an implicit contractive condition with an application," Fixed Point Theory and Applications, vol. 42, pp. 1-15, 2016.

[19] T. Abdeljawad, K. Ullah, and J. Ahmad, "Iterative algorithm for mappings satisfying $\left(B_{\gamma, \mu}\right)$ condition," Journal of Function Spaces, vol. 2020, Article ID 3492549, 7 pages, 2020.

[20] Z. Opial, "Weak convergence of the sequence of successive approximations for nonexpansive mappings," Bulletin of the American Mathematical Society, vol. 73, no. 4, p. 591, 1967.

[21] H. F. Senter and W. G. Dotson, "Approximating fixed points of nonexpansive mappings," Proceedings of the American Mathematical Society, vol. 44, no. 2, p. 375, 1974.

[22] J. A. Clarkson, "Uniformly convex spaces," Transactions of the American Mathematical Society, vol. 40, no. 3, pp. 396-414, 1936.

[23] W. Takahashi, Nonlinear Functional Analysis, Yokohoma Publishers, Yokohoma, Japan, 2000.

[24] R. P. Agarwal, D. O’Regan, and D. R. Sahu, "Fixed point theory for Lipschitzian-type mappings with applications series," Topological Fixed Point Theory and Its Applications, Springer, New York, NY, USA, 2009.

[25] J. Schu, "Weak and strong convergence to fixed points of asymptotically nonexpansive mappings," Bulletin of the Australian Mathematical Society, vol. 43, no. 1, p. 153, 1991.

[26] W. A. Kirk, "A fixed point theorem for mappings which do not increase distances," The American Mathematical Monthly, vol. 72, no. 9, p. 1004, 1965. 